\title{
Channel Tracking Using Particle Filtering in Unresolvable Multipath Environments
}

\author{
Tanya Bertozzi \\ Diginext, 45 Impasse de la Draille, 13857 Aix-en-Provence Cedex 3, France \\ Email: bertozzi@diginext.fr \\ Conservatoire National des Arts et Métiers (CNAM), 292 rue Saint-Martin, 75141 Paris Cedex 3, France
}

\section{Didier Le Ruyet}

Conservatoire National des Arts et Métiers (CNAM), 292 rue Saint-Martin, 75141 Paris Cedex 3, France

Email:leruyet@cnam.fr

\section{Cristiano Panazio}

Conservatoire National des Arts et Métiers (CNAM), 292 rue Saint-Martin, 75141 Paris Cedex 3, France Email:panazio.cristiano@cnam.fr

\author{
Han Vu Thien \\ Conservatoire National des Arts et Métiers (CNAM), 292 rue Saint-Martin, 75141 Paris Cedex 3, France \\ Email:vu-thien@cnam.fr
}

Received 1 May 2003; Revised 9 June 2004

\begin{abstract}
We propose a new timing error detector for timing tracking loops inside the Rake receiver in spread spectrum systems. Based on a particle filter, this timing error detector jointly tracks the delays of each path of the frequency-selective channels. Instead of using a conventional channel estimator, we have introduced a joint time delay and channel estimator with almost no additional computational complexity. The proposed scheme avoids the drawback of the classical early-late gate detector which is not able to separate closely spaced paths. Simulation results show that the proposed detectors outperform the conventional early-late gate detector in indoor scenarios.
\end{abstract}

Keywords and phrases: sequential Monte Carlo, multipath channels, importance sampling, timing estimation.

\section{INTRODUCTION}

In wireless communications, direct-sequence spread spectrum (DS-SS) techniques have received an increasing interest, especially for the third generation of mobile systems. In DS-SS systems, the adapted filter typically employed is the Rake receiver. This receiver is efficient to counteract the effects of frequency-selective channels. It is composed of fingers, each assigned to one of the most significant channel paths. The outputs of the fingers are combined proportionally to the power of each path for estimating the transmitted symbols (maximum-ratio combining). Unfortunately, the performance of the Rake receiver strongly depends on the quality of the estimation of the parameters associated with the channel paths. As a consequence, we have to estimate the delay of each path using a timing error detector (TED). This goal is generally achieved in two steps: acquisition and tracking. During the acquisition phase, the number and the delays of the most significant paths are determined. These delays are estimated within one half chip from the exact delays. Then, the tracking module refines the first estimation and follows the delay variations during the permanent phase. The conventional TED used during the tracking phase is the early-late gate-TED (ELG-TED) associated with each path. It is well known that the ELG-TED works very well in the case of a single fading path. However, in the presence of multipath propagation, the interference between the different paths can degrade its performance. In fact, the ELG-TED cannot separate the individual paths when they are closer than one chip period from the other paths, whereas a discrimination up to $T_{c} / 4$ can still increase the diversity of the receiver $\left(T_{c}\right.$ denotes the chip time) [1]. When the difference between the delays of two paths is contained in the interval $0-1.5 T_{c}$, we are in the presence of unresolvable multipaths. This scenario 
corresponds, for example, to the indoor scenario. The problem of unresolvable multipaths has recently been analyzed in $[2,3,4]$.

Particle filtering (PF) or sequential Monte Carlo (SMC) methods [5] represent the most powerful approach for the sequential estimation of the hidden state of a nonlinear dynamic model. The solution to this problem depends on the knowledge of the posterior probability density (PPD) of the hidden state given the observations. Except in a few special cases including linear Gaussian system models, it is impossible to analytically calculate a sequential expression of this PPD. It is necessary to adopt numerical approximations. The PF methods give a discrete approximation of the PPD of the hidden state by weighted points or particles which can be recursively updated as new observations become available.

The first main application of the PF methods was target tracking. More recently, these techniques have been successfully applied in communications, including blind equalization in Gaussian [6] and non-Gaussian [7, 8] noises and joint symbol and timing estimation [9]. For a complete survey of the communication problems dealt with using PF methods, see $[10]$.

In this paper we propose to use the PF methods for estimating the delays of the paths in multipath fading channels. Since these methods are based on a joint approach, they provide optimal estimates of the different channel delays. In this way, we can overcome the problem of the adjacent paths which causes the failure of the conventional single-path-tracking approaches in the presence of unresolvable multipaths. Moreover, we will combine the PF-based TED (PF-TED) with a conventional estimator for estimating the amplitudes of the channel coefficients. We will also apply the PF methods to the estimation of the channel coefficients in order to jointly estimate the delays and the coefficients.

This paper is organized as follows. In Section 2, we will introduce the system model. Then in Section 3, we will describe the conventional ELG-TED and the PF-TED. In Section 4, we will present the conventional estimators of the channel coefficients and the application of the PF methods to the joint estimation of the delays and the channel coefficients. In Section 5, we will give simulation results. Finally, we will draw a conclusion in Section 5.

\section{SYSTEM MODEL}

We consider a DS-SS system sending a complex data sequence $\left\{s_{n}\right\}$. The data symbols are spread by a spreading sequence $\left\{d_{m}\right\}_{m=0}^{N_{s}-1}$ where $N_{s}$ is the spreading factor.

The resulting baseband equivalent transmitted signal is given by

$$
e(t)=\sum_{n} s_{n} \sum_{m=0}^{N_{s}-1} d_{m} g\left(t-m T_{c}-n T\right)
$$

where $T_{c}$ and $T$ are respectively the chip and symbol period and $g(t)$ is the impulse response of the root-raised cosine filter with a rolloff factor equal to 0.22 in the case of the universal mobile telecommunications system (UMTS) [11].

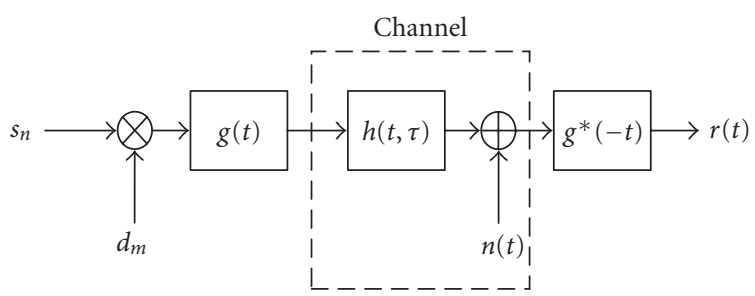

Figure 1: Equivalent lowpass transmission system model.

$h(t, \tau)$ denotes the overall impulse response of the multipath propagation channel with $L_{h}$ independent paths (widesense stationary uncorrelated scatterers (WSSUS) model):

$$
h(t, \tau)=\sum_{l=1}^{L_{h}} h_{l}(t) \delta\left(\tau-\tau_{l}(t)\right)
$$

Each path is characterized by its time-varying delay $\tau_{l}(t)$ and channel coefficient $h_{l}(t)$.

The signal at the output of the matched filter is given by

$$
r(t)=\sum_{l=1}^{L_{h}} h_{l}(t) \sum_{n} s_{n} \sum_{m=0}^{N_{s}-1} d_{m} R_{g}\left(t-m T_{c}-n T-\tau_{l}(t)\right)+\tilde{n}(t),
$$

where $\tilde{n}(t)$ represents the additive white gaussian noise (AWGN) $n(t)$ filtered by the matched filter and

$$
R_{g}(t)=\int_{-\infty}^{+\infty} g^{*}(\tau) g(t+\tau) d \tau
$$

is the total impulse response of the transmission and receiver filters.

Figure 1 shows the equivalent lowpass transmission model considered in this paper.

The output of the matched filter is used as the input of the Rake receiver. The Rake receiver model is shown in Figure 2. The Rake receiver is composed of $L$ branches corresponding to the $L$ most significant paths. In the $l$ th branch, the received and filtered signal $r(t)$ is sampled at time $m T_{c}+n T+\hat{\tau}_{l}$ in order to compensate the timing delay $\tau_{l}$ of the associated path with the estimate $\hat{\tau}_{l}$. The outputs of each branch are combined to estimate the transmitted symbols. The output of the Rake receiver is given as

$$
\hat{s}_{n}=\hat{s}(n T)=\frac{1}{N_{s}} \sum_{l=1}^{L} \hat{h}_{l}^{*} \sum_{m=0}^{N_{s}-1} d_{m}^{*} r\left(m T_{c}+n T+\hat{\tau}_{l}\right) .
$$

\section{THE TIMING ERROR DETECTION}

\subsection{The conventional TED}

The Rake receiver needs good timing delays and channel estimators for each path to extract the most signal power from the received signal and to maximize the signal-to-noise ratio at the output of Rake receiver. 


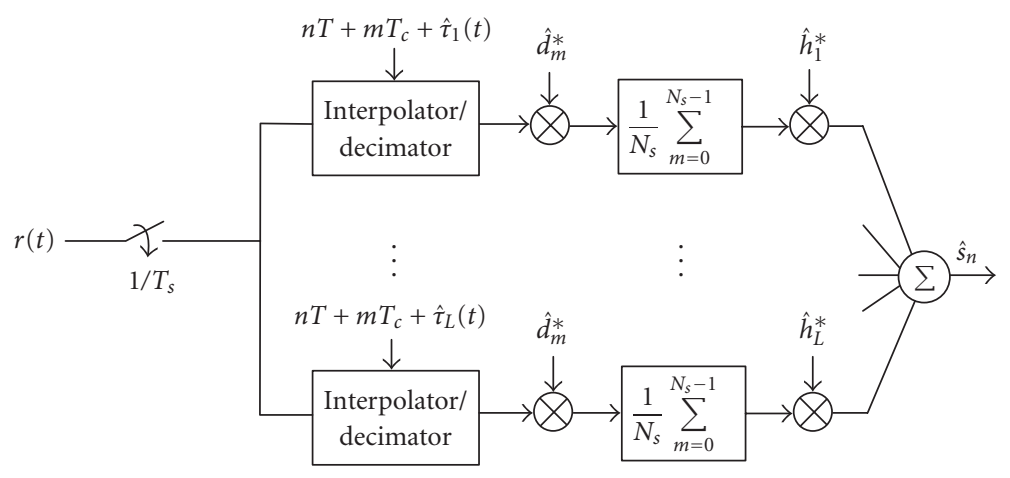

FIgURE 2: Rake receiver model.

The conventional TED for DS-SS systems is the ELGTED. The ELG-TED is devoted to the tracking of the delay of one path. It is composed of the early and late branches. The signal $r(t)$ is sampled at time $m T_{c}+n T+\hat{\tau}_{l} \pm \Delta$. In this paper, we will use $\Delta=T_{c} / 2$. We will restrict ourselves to the coherent ELG-TED where the algorithm uses an estimation of the transmitted data or the pilots when they are available. The output of a coherent ELG-TED associated with the $l$ th path is given by

$$
\begin{aligned}
& x_{n}= x(n T) \\
&=\operatorname{Re}\left\{\hat { s } _ { n } ^ { * } h _ { l } ^ { * } \sum _ { m = n N _ { s } } ^ { ( n + 1 ) N _ { s } - 1 } \left(r\left(m T_{c}+\hat{\tau}_{l}+\frac{T_{c}}{2}\right)\right.\right. \\
&\left.\left.\quad-r\left(m T_{c}+\hat{\tau}_{l}-\frac{T_{c}}{2}\right)\right) \hat{d}_{m}^{*}\right\} .
\end{aligned}
$$

The main limitation of the ELG-TED is its discrimination capability. Indeed, when the paths are unresolvable (separated by less than $T_{c}$ ), the ELG-TED is not able to correctly distinguish and track the path. This scenario corresponds for example to the indoor case.

These drawbacks motivated the proposed PF-TED.

\subsection{The PF-TED}

We propose to use the PF methods in order to jointly track the delay of each individual path of the channel. We assume that the acquisition phase has allowed us to determine the number of the most significant paths and to roughly estimate their delay.

The PF methods are used to sequentially estimate timevarying quantities from measures provided by sensors. In general, the physical phenomenon is represented by a state space model composed of two equations: the first describes the evolution of the unknown quantities called hidden state (evolution equation) and the second the relation between the measures called observations and the hidden state (observation equation). Given the initial distribution of the hidden state, the estimation of the hidden state at time $t$ based on the observations until time $t$ is known as Bayesian inference or Bayesian filtering. This estimation can be obtained through the knowledge of two distributions: the PPD of the sequence of hidden states from time 1 to time $t$ given the corresponding sequence of observations and the marginal distribution of the hidden state at time $t$ given the sequence of the observations until time $t$. Except in a few special cases including linear Gaussian state space models, it is impossible to analytically calculate these distributions. The PF methods provide a discrete and sequential approximation of the distributions. It can be updated when a new observation is available, without reprocessing the previous observations. The support of the distributions is discretized by particles, which are weighted samples evolving in time.

Tracking the delay of the individual channel paths can be interpreted as a Bayesian inference. The delays are the hidden state of the system and the model (3) of the received samples relating the observations to the delays represents the observation equation. We notice that this equation is nonlinear with respect to the delays and as a consequence, we cannot analytically estimate the delays. To overcome this nonlinearity, we propose to apply the PF methods.

The PF methods have previously been applied for the delay estimation in DS-CDMA systems $[12,13]$. In [12], the PF methods are used to jointly estimate the data, the channel coefficients, and the propagation delay. In [13], the PF methods are combined with a Kalman filter (KF) to respectively estimate the delay propagation and the channel coefficients; the information symbols are assumed known, provided by a Rake receiver. In both papers, the delays of each channel path are considered known and multiple of the sampling time; therefore, only the propagation delay is estimated. In this paper, the approach is different. We suppose that each channel path has a slow time-varying delay, unknown at the receiver. This environment can represent an indoor wireless communication. We assume that the information symbols are known or have been estimated essentially for three reasons:

(i) the computational complexity of the receiver should be reduced;

(ii) the channel estimation is typically performed transmitting known pilot symbols, for example using a specific channel as the common pilot channel $(\mathrm{CPICH})$ of the UMTS; 


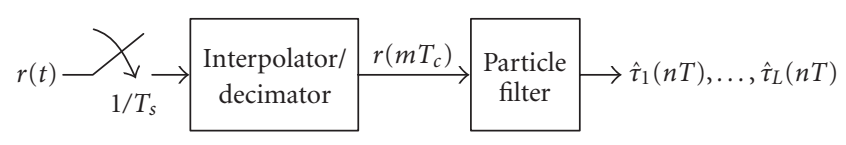

FIgURE 3: Structure of the proposed PF-TED.

(iii) the PF methods applied to the estimation of the information symbols perform slightly worse than simple deterministic algorithms $[12,14]$.

Firstly, we will apply the PF methods only to the estimation of the delays of each channel path, considering that the channel coefficients are known. In the next paragraph, we will introduce the estimation of the channel coefficients.

The structure of the proposed PF-TED is shown in Figure 3. This estimator operates on samples from the matched filter output taken at an arbitrary sampling rate $1 / T_{s}$ (at least Nyquist sampling). Then, the samples are processed by means of interpolation and decimation in order to obtain intermediate samples at the chip rate $1 / T_{c}$. These samples are the input of the particle filter. In order to reduce the computational complexity of the PF-TED and since the time variation of the delays is slow with respect to the symbol duration, we choose that the particle filter works at the symbol rate $1 / T$. Moreover, in order to exploit all the information contained in the chips of a symbol period, the equations of the PF algorithm are modified. The PF algorithm proposed in this paper is thus the adaptation of the PF methods to a DS-SS system.

Following [15], the evolution of the delays of the channel paths can be described as a first-order autoregressive (AR) process:

$$
\begin{gathered}
\tau_{1, n}=\alpha_{1} \tau_{1, n-1}+v_{1, n}, \\
\vdots \\
\tau_{L, n}=\alpha_{L} \tau_{L, n-1}+v_{L, n},
\end{gathered}
$$

where $\tau_{l, n}$ for $l=1, \ldots, L$ denotes the delay of the $l$ th channel path at time $n, \alpha_{1}, \ldots, \alpha_{L}$ express the possible time variation of the delays from a time to the next one, and $v_{1}, \ldots, v_{L}$ are AWGN with zero mean and variance $\sigma_{v}^{2}$. Note that the time index $n$ is an integer multiple of the symbol duration.

The estimation of the delays can be achieved using the minimum mean square error (MMSE) method or the maximum a posteriori (MAP) method. The MMSE solution is given by the following expectation:

$$
\hat{\tau}_{n}=E\left[\tau_{n} \mid r_{1: n}\right]
$$

where $\tau_{n}=\left\{\tau_{1, n}, \ldots, \tau_{L, n}\right\}$ and $r_{1: n}$ is the sequence of received samples from time 1 to $n$. The calculation of (8) involves the knowledge of the marginal distribution $p\left(\tau_{n} \mid r_{1: n}\right)$. Unlike the MMSE solution that yields an estimate of the delays at each time, the MAP method provides the estimate of the hidden state sequence $\tau_{1: n}=\left\{\tau_{1}, \ldots, \tau_{n}\right\}$ :

$$
\hat{\tau}_{1: n}=\arg \max _{\tau_{1: n}} p\left(\tau_{1: n} \mid r_{1: n}\right) .
$$

The calculation of (9) requires the knowledge of the PPD $p\left(\tau_{1: n} \mid r_{1: n}\right)$.

The simulations give similar results for the MMSE method and the MAP method. Hence, we choose to adopt the MMSE solution as in [9]. In order to obtain samples from the marginal distribution, we use the sequential importance sampling (SIS) approach [16]. Applying the definition of the expectation, (8) can be expressed as follows:

$$
\hat{\tau}_{n}=\int \tau_{n} p\left(\tau_{n} \mid r_{1: n}\right) d \tau_{n}
$$

The aim of the SIS technique is to approximate the marginal distribution $p\left(\tau_{n} \mid r_{1: n}\right)$ by means of weighted particles:

$$
p\left(\tau_{n} \mid r_{1: n}\right) \approx \sum_{i=1}^{N_{p}} \tilde{w}_{n}^{(i)} \delta\left(\tau_{n}-\tau_{n}^{(i)}\right)
$$

where $N_{p}$ is the number of particles, $\tilde{w}_{n}^{(i)}$ is the normalized importance weight at time $n$ associated with the particle $i$, and $\delta\left(\tau_{n}-\tau_{n}^{(i)}\right)$ denotes the Dirac delta centered in $\tau_{n}=\tau_{n}^{(i)}$.

The phases of the PF-TED based on the SIS approach are summarized below.

(1) Initialization. In this paper, we apply the PF methods for the tracking phase, assuming that the number of the channel paths and the initial value of the delay for each path have been estimated during the acquisition phase [17]. We assume that the error on the delay estimated by the acquisition phase belongs to the interval $\left(-T_{c} / 2, T_{c} / 2\right)$. Hence, the a priori probability density $p\left(\tau_{0}\right)$ can be considered uniformly distributed in $\left(\hat{\tau}_{0}-T_{c} / 2, \hat{\tau}_{0}+T_{c} / 2\right)$, where $\hat{\tau}_{0}$ is the delay provided by the acquisition phase. Note that the PF methods can be used also for the acquisition phase. However, the number of particles has to be increased, because we have no a priori information on the initial value of the delays.

(2) Importance sampling. The time evolution of the particles is achieved with an importance sampling distribution. When $r_{n}$ is observed, the particles are drawn according to the importance function. In general, the importance function is chosen to minimize the variance of the importance weights associated with each particle. In fact, it can be shown that the variance of the importance weights can only increase stochastically over time [16]. This means that, after a few iterations of the SIS algorithm, only one particle has a normalized weight almost equal to 1 and the other weights are very close to zero. Therefore, a large computational effort is devoted to updating paths with almost no contribution to the final estimate. In order to avoid this behavior, a resampling phase of the particles is inserted among the recursions of the SIS algorithm. To limit this degeneracy phenomenon, we need to use the optimal importance function [16], given by

$$
\pi\left(\tau_{n}^{(i)} \mid \tau_{1: n-1}^{(i)}, r_{1: n}\right)=p\left(\tau_{n}^{(i)} \mid \tau_{n-1}^{(i)}, r_{n}\right)
$$


Unfortunately, the optimal importance function can be analytically calculated only in a few cases, including the class of models represented by a Gaussian state space model with linear observation equation. In this case, the observation equation (3) is nonlinear and thus, the optimal importance function cannot be analytically determined. We can consider two solutions to this problem [16]:

(i) the a priori importance function $p\left(\tau_{n}^{(i)} \mid \tau_{n-1}^{(i)}\right)$;

(ii) an approximated expression of the optimal importance function by linearization of the observation equation about $\tau_{l, n}^{(i)}=\alpha_{l} \tau_{l, n-1}^{(i)}$ for $l=1, \ldots, L$.

Since the second solution involves the derivative calculation of the nonlinear observation equation, and hence very complex operations, we choose the a priori importance function as in [9]. Considering that the noises $v_{l, n}$ for $l=1, \ldots, L$ in (7) are Gaussian, the importance function for each delay $l$ is a Gaussian distribution with mean $\alpha_{l} \tau_{l, n-1}^{(i)}$ and variance $\sigma_{v}^{2}$.

(3) Weight update. The evaluation of the importance function for each particle at time $n$ enables the calculation of the importance weights [16]:

$$
w_{n}^{(i)}=w_{n-1}^{(i)} \frac{p\left(r_{n} \mid \tau_{n}^{(i)}\right) p\left(\tau_{n}^{(i)} \mid \tau_{n-1}^{(i)}\right)}{\pi\left(\tau_{n}^{(i)} \mid \tau_{1: n-1}^{(i)}, r_{1: n}\right)}
$$

This expression represents the calculation of the importance weights if we only consider the samples of the received signal at the symbol rate. However, in a DS-SS system we have additional information provided by $N_{s}$ samples for each symbol period due to the spreading sequence. Consequently, we modify (13) taking into account the presence of a spreading sequence. Indeed, observing that the received samples are independent, the probability density $p\left(r_{n} \mid \tau_{n}^{(i)}\right)$ at the symbol rate can be written as

$$
p\left(r_{n} \mid \tau_{n}^{(i)}\right)=\prod_{m=n N_{s}}^{(n+1) N_{s}-1} p\left(r_{m} \mid \tau_{n}^{(i)}\right)
$$

Considering (3) at the chip rate and recalling the assumptions of known symbols, the probability density $p\left(r_{m} \mid \tau_{n}^{(i)}\right)$ is Gaussian. Typically, the received sample $r_{m}$ is complex. For the calculation of the Gaussian distribution, we can write $r_{m}$ as a bidimensional vector with components being the real part and the imaginary part of $r_{m}$. The probability density $p\left(r_{m} \mid \tau_{n}^{(i)}\right)$ is thus given by

$$
p\left(r_{m} \mid \tau_{n}^{(i)}\right)=\frac{1}{\pi \sigma_{n}^{2}} \exp \left\{-\frac{1}{\sigma_{n}^{2}}\left|r_{m}-\mu_{m}^{(i)}\right|^{2}\right\},
$$

where $\sigma_{n}^{2}$ is the variance of the AWGN $\tilde{n}(t)$ in (3) and the mean $\mu_{m}^{(i)}$ is obtained by

$$
\mu_{m}^{(i)}=\sum_{l=1}^{L} h_{l, n} s_{n} \sum_{k=m-3}^{m+3} d_{k} R_{g}\left(m T_{c}-k T_{c}-n T-\tau_{l, n}^{(i)}\right)
$$

In order to reduce the computational complexity of the PFTED, in (16) we have assumed that the contribution of the raised cosine filter $R_{g}$ to the sum on the spreading sequence is limited to the previous 3 and next 3 samples. By substitution of (15) in (14), the latter becomes

$$
p\left(r_{n} \mid \tau_{n}^{(i)}\right)=\left(\frac{1}{\pi \sigma_{n}^{2}}\right)^{N_{s}} \exp \left\{-\frac{1}{\sigma_{n}^{2}} \sum_{m=n N_{s}}^{(n+1) N_{s}-1}\left|r_{m}-\mu_{m}^{(i)}\right|^{2}\right\} .
$$

Assuming the a priori importance function, (13) yields

$$
\begin{aligned}
w_{n}^{(i)} & =w_{n-1}^{(i)} p\left(r_{n} \mid \tau_{n}^{(i)}\right) \\
& =w_{n-1}^{(i)}\left(\frac{1}{\pi \sigma_{n}^{2}}\right)^{N_{s}} \exp \left\{-\frac{1}{\sigma_{n}^{2}} \sum_{m=n N_{s}}^{(n+1) N_{s}-1}\left|r_{m}-\mu_{m}^{(i)}\right|^{2}\right\} .
\end{aligned}
$$

Finally, the importance weights in (18) are normalized using the following expression:

$$
\tilde{w}_{n}^{(i)}=\frac{w_{n}^{(i)}}{\sum_{j=1}^{N_{p}} w_{n}^{(j)}} .
$$

(4) Estimation. By substitution of (11) into (10), we obtain at each time the MMSE estimate:

$$
\hat{\tau}_{n}=\sum_{i=1}^{N_{p}} \tilde{w}_{n}^{(i)} \tau_{n}^{(i)} .
$$

(5) Resampling. This algorithm presents a degeneracy phenomenon. After a few iterations of the algorithm, only one particle has a normalized weight almost equal to 1 and the other weights are very close to zero. This problem of the SIS method can be eliminated with a resampling of the particles. A measure of the degeneracy is the effective sample size $N_{\text {eff }}$, estimated by

$$
\hat{N}_{\text {eff }}=\frac{1}{\sum_{i=1}^{N_{p}}\left(\tilde{w}_{n}^{(i)}\right)^{2}} .
$$

When $\hat{N}_{\text {eff }}$ is below a fixed threshold $N_{\text {thres}}$, the particles are resampled according to the weight distribution [16]. After each resampling task, the normalized weights are initialized to $1 / N_{p}$.

\section{THE ESTIMATION OF THE CHANNEL COEFFICIENTS}

\subsection{The conventional estimators}

Channel estimation is performed using the known pilot symbols. If we suppose that the channel remains almost unchanged during the slot, the conventional estimator of the channel coefficients of the $l$ th path is obtained by correlation using the known symbols [18]:

$$
\hat{h}_{l}=\frac{1}{N_{\text {pilot }} N_{s}} \sum_{n=0}^{N_{\text {pilot }}-1} \sum_{m=0}^{N_{s}-1} s_{n}^{*} d_{m}^{*} r\left(m T_{c}+n T+\hat{\tau}_{l, n}\right),
$$


where $N_{\text {pilot }}$ is the number of pilots in a slot. For each path, the received signal is sampled at time $m T_{c}+n T+\hat{\tau}_{l, n}$ in order to compensate its delay. Then the samples are multiplied by the despread sequence and summed on the whole sequence of pilot symbols. The problem of this estimator is that when the delays are unresolvable, the estimation becomes biased. To eliminate this bias, we can use an estimator based on the maximum likelihood (ML) criterion. In $[1,19]$, a simplified version of the ML estimation is proposed. The channel coefficients which maximize the ML criterion are given by

$$
\hat{\mathbf{h}}=\mathbf{P}^{-1} \mathbf{a},
$$

where $\hat{\mathbf{h}}=\left(\hat{h}_{1}, \ldots, \hat{h}_{L}\right), \mathbf{P}$ is an $L \times L$ matrix with elements $P_{i j}=R_{g}\left(\tau_{i, n}-\tau_{j, n}\right)$, and $\mathbf{a}$ is the vector of the channel coefficients calculated using (22).

\subsection{The PF-based joint estimation of the delays and the channel coefficients}

We can apply the PF methods to jointly estimate the delays of each path and the channel coefficients with a very low additional cost in terms of computational complexity. This is a suboptimal solution, since the observation equation (3) is linear and Gaussian with respect to the channel coefficients. The optimal solution is represented by a KF. However, combining the PF methods and the KF to jointly estimate the delays and the channel coefficients involves the implementation of a KF. It is better to use the particles employed for the delay estimation and to associate to each particle the estimation of the channel coefficients.

In this case, the hidden state is composed of the $L$ delays and the $L$ channel coefficients of each individual path. When a particle evolves in time, its new position is thus determined by the evolution of the delays and the evolution of the channel coefficients. The delays evolve as described for the PF-TED. For the channel coefficients, we assume that the time variations are slow as, for example, in indoor environments. Hence, the evolution of the channel coefficients can be expressed by the following first-order AR model:

$$
\begin{gathered}
h_{1, n}=\beta_{1} h_{1, n-1}+z_{1, n}, \\
\vdots \\
h_{L, n}=\beta_{L} h_{L, n-1}+z_{L, n},
\end{gathered}
$$

where $\beta_{1}, \ldots, \beta_{L}$ describe the possible time variation of the channel coefficients from a time to the next one and $z_{1}, \ldots, z_{L}$ are AWGN with zero mean and variance $\sigma_{z}^{2}$. The parameters of the channel AR model (24) are chosen according to the Doppler spread of the channel [20]. Notice that this joint estimator operates at the symbol rate as the PF-TED.

As for the delays, we only consider the MMSE method for the estimation of the channel coefficients and we use the a priori importance function:

$$
\pi\left(h_{n}^{(i)} \mid h_{1: n-1}^{(i)}, r_{1: n}\right)=p\left(h_{n}^{(i)} \mid h_{n-1}^{(i)}\right),
$$

where $h_{n}=\left\{h_{1, n}, \ldots, h_{L, n}\right\}$. Considering that the noises $z_{l, n}$ for $l=1, \ldots, L$ in $(24)$ are Gaussian, the importance function for the channel coefficients is a Gaussian distribution with mean $\beta_{l} h_{l, n-1}^{(i)}$ and variance $\sigma_{z}^{2}$. To determine the positions of the particles at time $n$ from the positions at time $n-1$, each particle is drawn according to $p\left(\tau_{n}^{(i)} \mid \tau_{n-1}^{(i)}\right)$ and (25).

The calculation of the importance weights is very similar to the case of the PF-TED. The only difference is that the channel coefficients $h_{l, n}$ are replaced by the support of the particles $h_{l, n}^{(i)}$ to calculate the mean (16).

\section{SIMULATION RESULTS}

In this section, we will compare the performance of the conventional ELG-TED and the PF-TED. In order to demonstrate the gain achieved using the latter, we will consider different indoor scenarios with a two-path Rayleigh channel with the same average power on each path and a maximum Doppler frequency of $19 \mathrm{~Hz}$ corresponding to a mobile speed of $10 \mathrm{Km} / \mathrm{h}$ for a carrier frequency of $2 \mathrm{GHz}$. The simulation setup is compatible with the UMTS standard. In these conditions, the time variations of the channel delays can be expressed by the model (7), with $\alpha_{1}=\cdots=\alpha_{L}=0.99999$ and $\sigma_{v}^{2}=10^{-5}$ [15]. Moreover, the time variations of the channel coefficients can be represented by the model (24), $\beta_{1}=\cdots=\beta_{L}=0.999$ and $\sigma_{z}^{2}=10^{-3}$.

In these simulations, a CPICH is used. In each slot of $\mathrm{CPICH}, 40$ pilot symbols equal to 1 are expanded into a chip level by a spreading factor of 64 . The spreading sequence is a $\mathrm{PN}$ sequence changing at each symbol.

\subsection{Tracking performance}

We assume that the channel coefficients are known to evaluate the TED's tracking capacity and the simulation time is equal to 0.333 second, corresponding to 500 slots. We have firstly considered the delays of the two paths varying according to the following model:

$$
\begin{aligned}
& \tau_{1, n}=\alpha_{1} \tau_{1, n-1}+v_{1, n}, \\
& \tau_{2, n}=\alpha_{2} \tau_{2, n-1}+v_{2, n},
\end{aligned}
$$

where $\alpha_{1}=\alpha_{2}=0.999, \sigma_{v, 1}^{2}=\sigma_{v, 2}^{2}=0.001, \tau_{1,0}=0$, and $\tau_{2,0}=1$.

Figure 4 shows one realization of the considered delays and the tracking performance of two ELG-TEDs used for the estimation of the two delays. We assume that $E_{s} / N_{0}=10 \mathrm{~dB}$, where $E_{s}$ is the energy per symbol and $N_{0}$ is the unilateral spectral power density. The classical ELG-TED presents difficulties to follow the time variation of the two delays, especially when the delay separation becomes less than $1 T_{c}$.

However, it is very important for the TED to distinguish the different paths of the channel to enable the Rake receiver to exploit the diversity contained in the multipath nature of the channel. In [1], it has been shown that the gain in diversity decreases as the separation between the paths decreases. In particular, a loss of $2.5 \mathrm{~dB}$ in the performance of the matched filter bound for a BER equal to $10^{-2}$, passing 


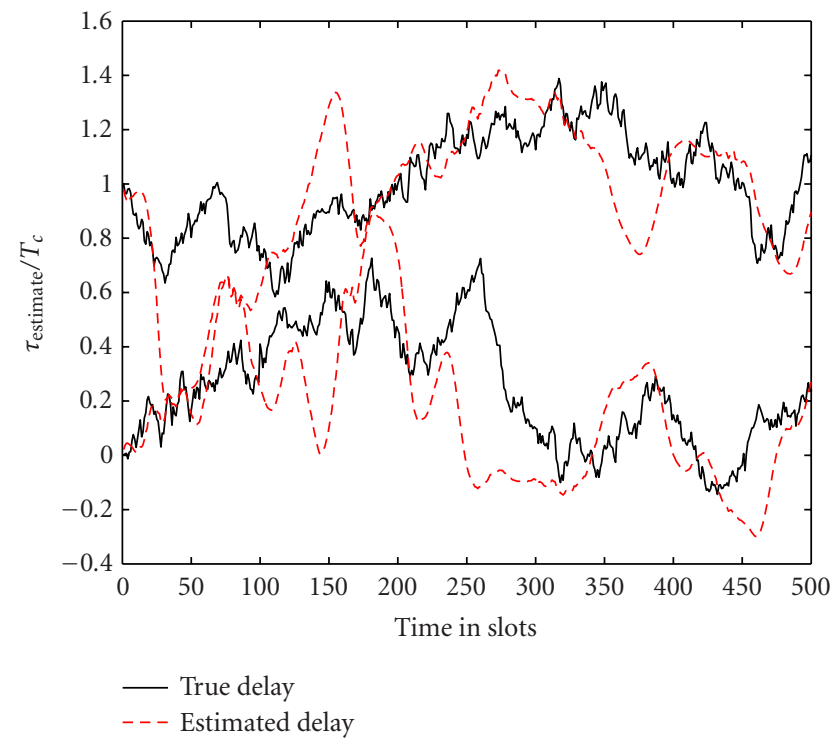

FIGURE 4: Delay tracking with the conventional ELG-TED.

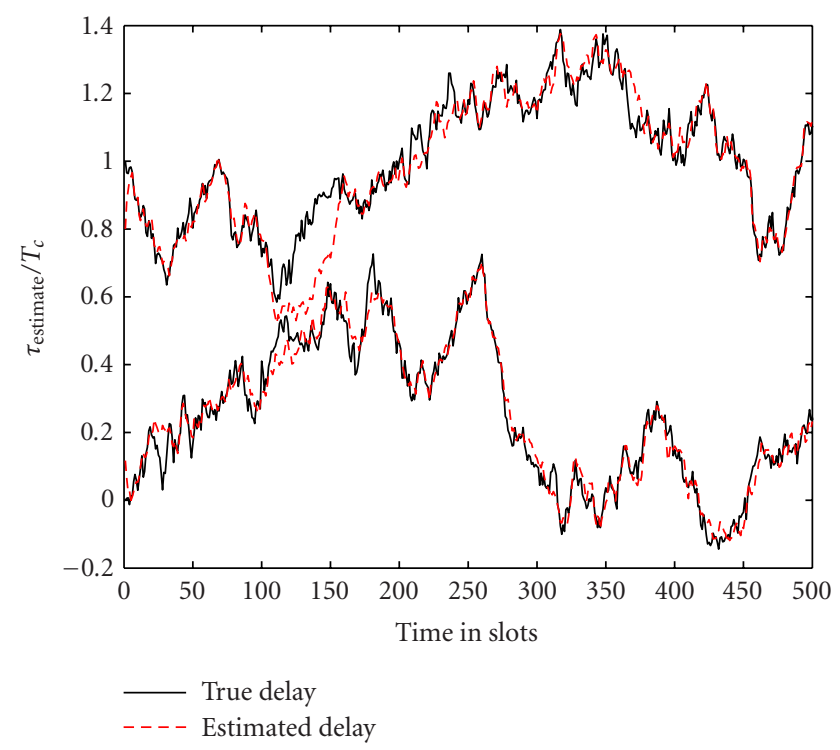

FIGURE 5: Delay tracking with the PF-TED.

from $T_{c}$ to $T_{c} / 4$, has been observed. Moreover, it has been noted that an interesting gain in diversity occurs if the TED distinguishes paths separated by more than $T_{c} / 4$. On the other hand, it has been found that the performance of the matched filter bound for a separation of $T_{c} / 8$ is very close to the one obtained with only one path. Consequently, the TED discrimination capacity has to be equal to $T_{c} / 4$. Unfortunately, the ELG-TED fails to distinguish all the paths with a delay separation less than $1 T_{c}$. In Figure 5, we can observe how the discrimination capacity of the TED can be improved using the PF methods.

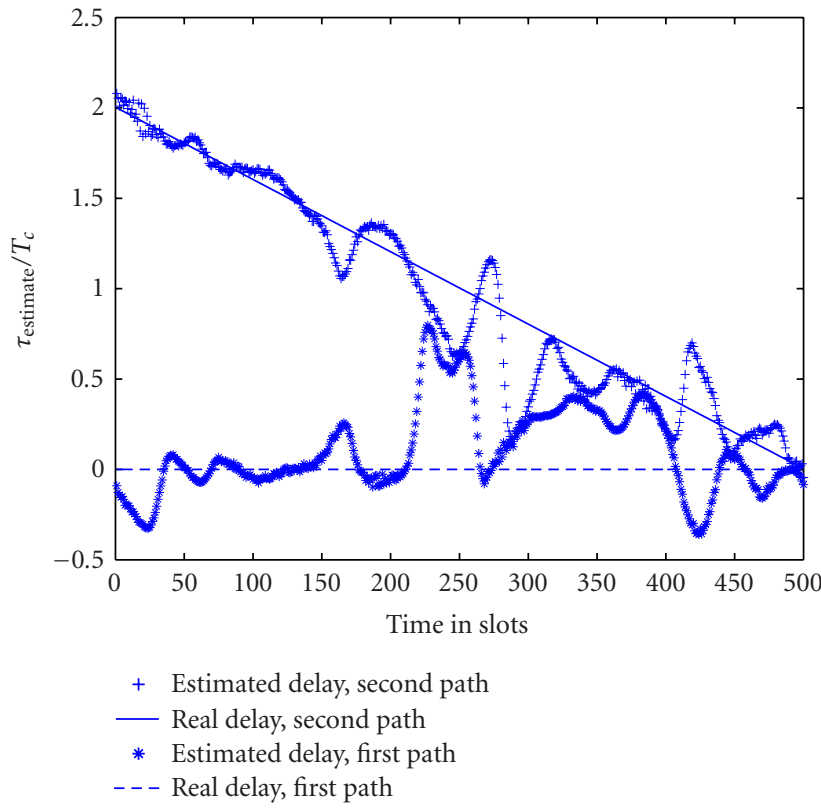

FIgURE 6: Delay tracking with the conventional ELG-TED.

In order to better highlight this behavior, we have fixed the delay of the first path at 0 and the delay of the second path is decreasing linearly from $2 T_{c}$ to 0 over a simulation time of 0.333 second corresponding to 500 slots. We assume that $E_{s} / N_{0}=10 \mathrm{~dB}$, where $E_{s}$ is the energy per symbol and $N_{0}$ is the unilateral spectral power density.

Firstly, we consider that the channel coefficients are known to evaluate the TED's tracking capacity. Figure 6 gives a representative example of the evolution of the two estimated delays using two ELG-TEDs. As soon as the difference between the two delays is lower than $1 T_{c}$, due to the correlation between the two paths, the estimated delays tend to oscillate around each real delay. The ELG-TEDs are no longer able to perform the correct tracking of the delays. On the other hand, as shown in Figure 7, the proposed PF-TED is able to track almost perfectly the two paths. These results have been obtained using a particle filter with only 10 particles.

Then, we have introduced the estimation of the channel coefficients into the TED. Figure 8 shows the results obtained with two ELG-TEDs combined with the conventional estimator based on the correlation. As soon as the difference between the two delays is lower than $1 T_{c}$, the detectors no longer recognize the two paths: the weaker path merges with the stronger one.

In Figure 9, the PF-TED is also associated with the conventional estimator of the channel coefficients based on the correlation. When the delay of the second path becomes less than $1 T_{c}$, the channel estimator decreases its capacity to track the time variations of the channel coefficients and the PF-TED cannot track the delays of the two paths. To improve the channel estimation, we associate the PF-TED with the ML estimator, as shown in Figure 10. In this case, the PF-TED can track the delay of the second path up to $T_{c} / 2$. 


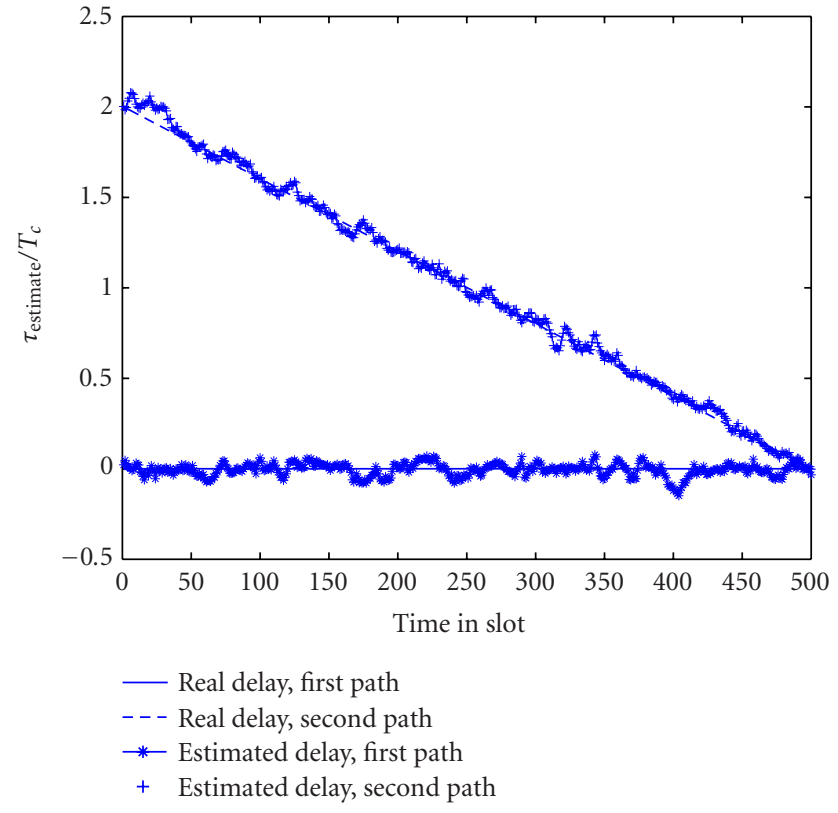

Figure 7: Delay tracking with the PF-TED.

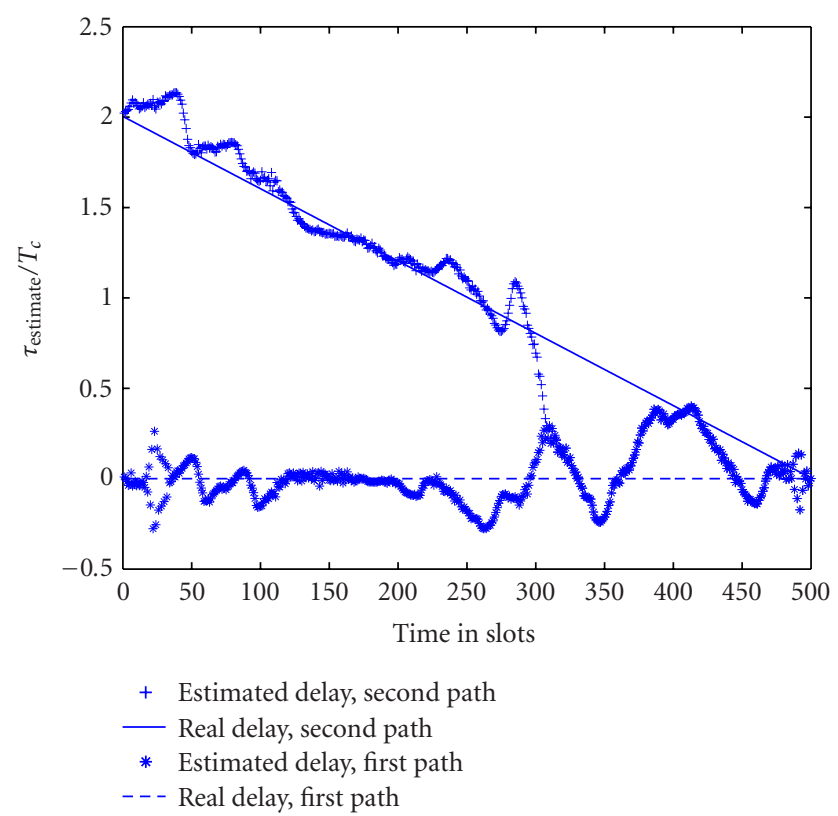

FIGURE 8: Delay tracking with the conventional ELG-TED associated with a conventional channel coefficient estimator based on the correlation.

For smaller delays, the PF-TED continues to distinguish the two paths, but it cannot follow the time variations of the second delay. The delay of the second path remains close to the values estimated at $T_{c} / 2$.

Using the PF methods to jointly estimate the delays and the channel coefficients, we can notice in Figure 11 that the PF-TED can track the time variations of the second path. This solution implies only a low additional cost in terms of

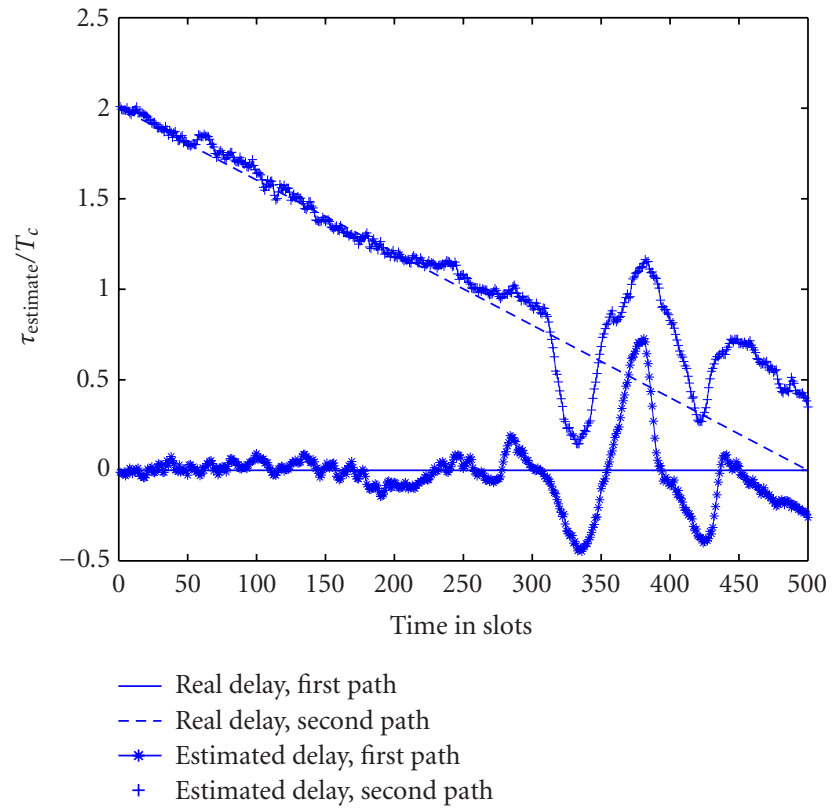

FIgure 9: Delay tracking with the PF-TED associated with a conventional channel coefficient estimator based on the correlation.

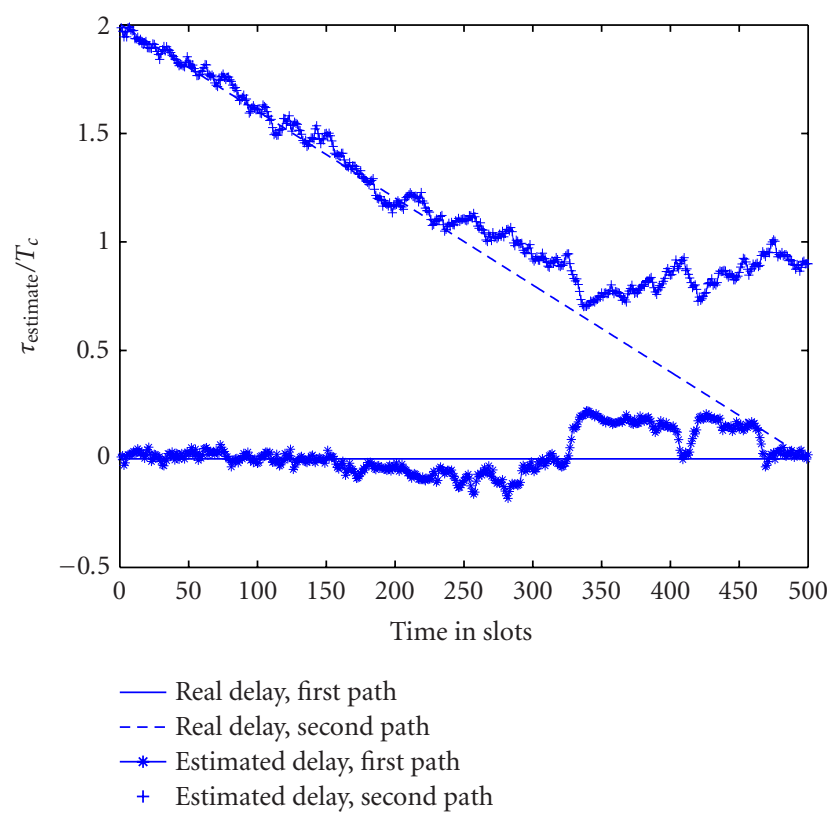

Figure 10: Delay tracking with the PF-TED associated with a conventional channel coefficient estimator based on the ML.

computational complexity with respect to the PF-TED, since it exploits the set of particles used for the delay estimation for the channel coefficient estimation.

\subsection{Mean square error of the delay estimators}

In this section, we will compare the estimation of the mean square error (MSE) estimating $\tau_{n}$ of the ELG-TED and the PF-TED with the lower posterior Cramer-Rao bound 


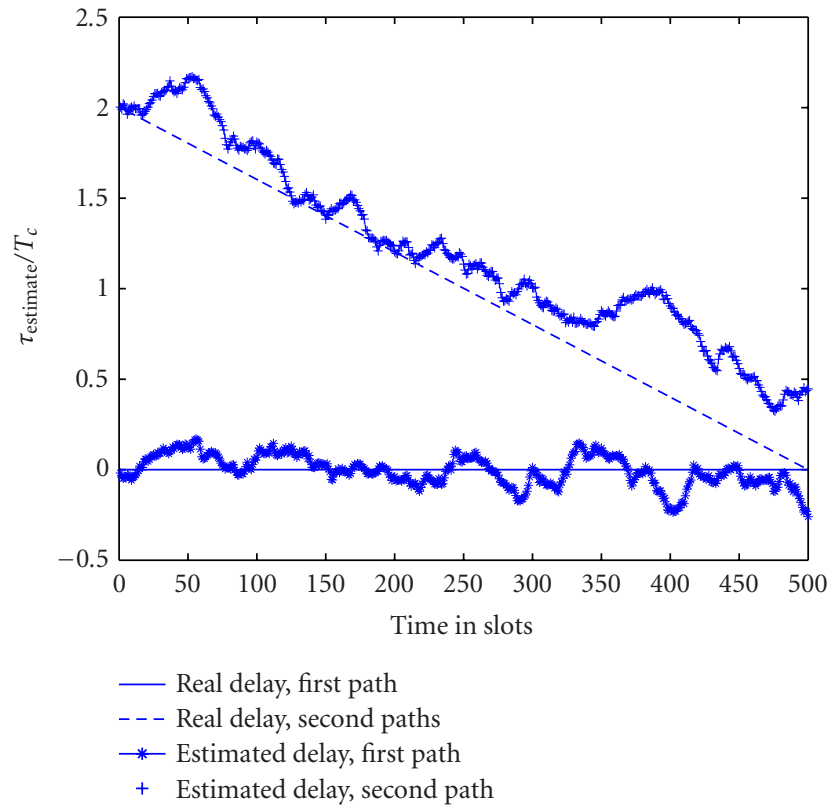

FIGURE 11: Delay tracking with a joint delay and channel coefficient estimator based on the PF methods.

(PCRB). In the Bayesian context of this paper, the PCRB [21] is more suitable than the Cramer-Rao bound [22] to evaluate the MSE of varying unknown parameters.

The PCRB for estimating $\tau_{n}$ using $r_{1: n}$ has the form

$$
E\left(\hat{\tau}_{n}-\tau_{n}\right)^{2} \geq J_{n, n}^{-1}
$$

where $J_{n, n}$ is the right lower element of the $n \times n$ Fisher information matrix.

In [21], the authors have shown how to recursively evaluate $J_{n, n}$. For our application, the nonlinear filtering system is

$$
\begin{gathered}
\tau_{n+1}=\alpha \tau_{n}+v_{n}, \\
r_{n}=z_{n}\left(\tau_{n}\right)+\tilde{n}_{n},
\end{gathered}
$$

where the second relation represents the nonlinear observation equation (3) at chip rate.

Since the spreading sequence is different at each chip time, we have to evaluate $z_{n}\left(\tau_{n}\right)$ at this rate.

From the general recursive equation given in [21], the sequence $\left\{J_{n, n}\right\}$ can be obtained as follows:

$$
\begin{aligned}
J_{n+1, n+1}= & \sigma_{v}^{-1}+E\left[\nabla_{\tau_{n+1}} z_{n+1}\left(\tau_{n+1}\right)\right]^{2} \sigma_{n}^{-1} \\
& -\left(\alpha \sigma_{v}^{-1}\right)^{2}\left(J_{n, n}+\alpha^{2} \sigma_{v}^{-1}\right)^{-1} .
\end{aligned}
$$

In order to calculate $E\left[\nabla_{\tau_{n+1}} z_{n+1}\left(\tau_{n+1}\right)\right]$, we have applied a Monte Carlo evaluation. We generate $M$ i.i.d. state trajectories of a given length $N_{t}\left\{\tau_{0}^{i}, \tau_{1}^{i}, \ldots, \tau_{N_{t}}^{i}\right\}$ with $1 \leq i \leq M$ by simulating the system model defined in (28) starting from an initial state $\tau_{0}$ drawn from the a priori probability density $p\left(\tau_{0}\right)$. For the calculation, we fixed $M=100$.

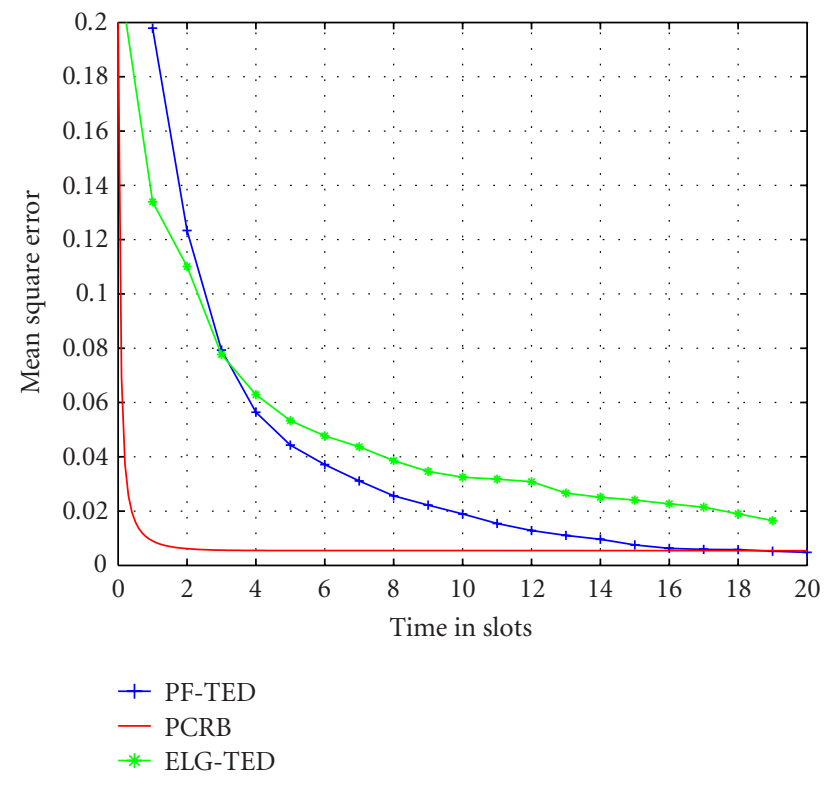

FIGURE 12: Comparison of the PCRB with the MSE estimating $\tau_{n}$ of ELG-TED and PF-TED.

In Figure 12, we show the comparison of the PCRB with the MSE estimating $\tau_{n}$ of the ELG-TED and PF-TED. For both algorithms, we use a uniform initial pdf $p\left(\tau_{0}\right)$. For the PF-TED, the 10 particles were initialized uniformly in the interval $\left\{-T_{c} / 2, T_{c} / 2\right\}$. The signal-to-noise ratio $E_{s} / N_{0}$ was fixed to $10 \mathrm{~dB}$. We can see in Figure 12 that the PF-TED outperforms the ELG-TED and reaches the PCRB bound after 15 slots. The slow convergence of the ELG-TED and PF-TED compared to the PCRB can be explained since the two TEDs are updated at each symbol while the PCRB bound is calculated for each chip.

\subsection{Performance evaluation}

Figure 13 shows the BERs versus $E_{s} / N_{0}$ considering a twopath channel with the same average power on each path. The delays of the first and second paths were respectively fixed at 0 and $1 T_{c}$. The same maximum Doppler frequency as above was used. The BER values have been averaged over 50000 bits.

When using two ELG-TEDs, except when the channel is known, the performance is very poor compared to the maximum achievable performance (known delays and channel coefficients). On the other hand, the PF-TED with channel coefficients known or estimated reaches the optimal performance. We can conclude that the considered TED must be able to separate the different paths of the channel, otherwise the performance of the Rake receiver breaks down.

\section{CONCLUSIONS}

In this paper we have proposed to use the PF methods in order to track the delay of the different channel paths. We have assumed that an acquisition phase has already provided an initial estimation of these delays. 


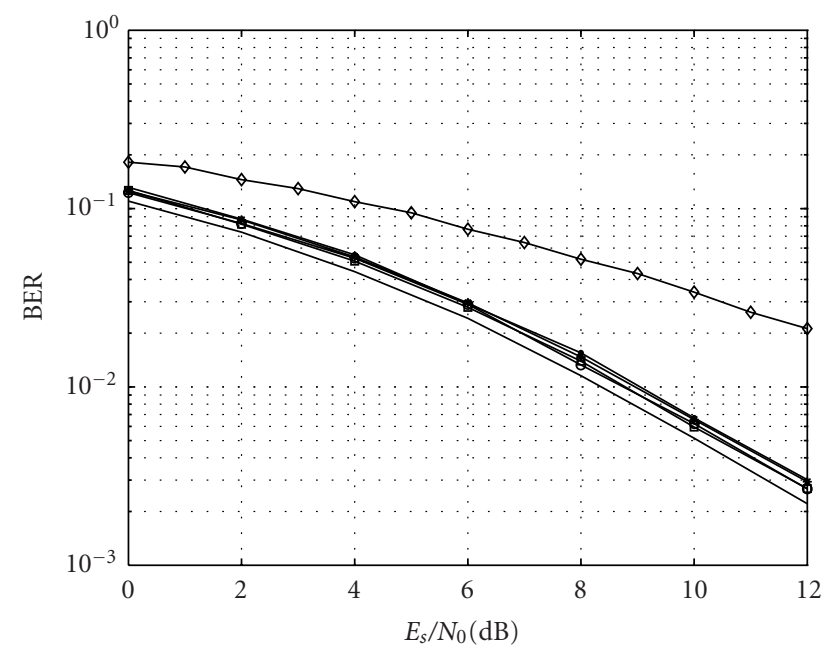

$$
\begin{aligned}
& \text { — Rake known delay, known channel } \\
& \rightarrow \text { ELG-TED known channel } \\
& \bullet \text { PF-TED known channel } \\
& \smile \text { ELG-TED estimated channel correlation } \\
& \rightarrow \text { PF-TED estimated channel correlation } \\
& \multimap \text { PF-TED estimated channel PF }
\end{aligned}
$$

Figure 13: Performance comparison of the ELG-TED and the PFTED.

We have firstly considered that the channel coefficients are known. We have compared the tracking capacity of the conventional ELG-TED and the proposed PF-TED. We have shown that when the delays of the channel paths become very close (less than $1 T_{c}$ ), the ELG-TED is unable to track the time variations of the delays. However, the PF-TED continues to track the delays.

We have introduced the channel coefficient estimation to the TED. We have considered two classical methods: the estimation based on the correlation using pilot symbols and the estimation based on the ML criterion. We have shown that the ELG-TED with estimation of the channel coefficients loses the capacity to distinguish the paths when the delays are very closed. On the other hand, the PF-TED associated with the classical two-channel estimator is able to separate the different paths. However, for very close delays the channel estimators prevent the PF-TED from tracking the time variations of the delays. We have proposed to estimate jointly the delays and the channel coefficients using the PF methods to avoid this loss of tracking. We have found that the joint estimation enables a better tracking of the delays.

Finally, we have seen that it is very important for the Rake receiver that the TED can distinguish the different paths of the channel. We have observed that in the case of unresolvable paths, the ELG-TED confuses the paths and the performance of the Rake receiver is very poor.

As a conclusion, we can say that the PF-TED based on the joint estimation of the delays and the channel coefficients can be a good substitute of the classical ELG-TED, specially for indoor wireless communications. Moreover, the computational complexity of the PF-TED is very limited, since we have used only 10 particles.

\section{REFERENCES}

[1] H. Boujemaa and M. Siala, "Rake receivers for direct sequence spread spectrum systems," Ann. Telecommun., vol. 56, no. 5-6, pp. 291-305, 2001.

[2] V. Aue and G. P. Fettweis, "A non-coherent tracking scheme for the RAKE receiver that can cope with unresolvable multipath," in Proc. IEEE International Conference on Communications (ICC '99), pp. 1917-1921, Vancouver, BC, Canada, June 1999.

[3] R. De Gaudenzi, "Direct-sequence spread-spectrum chip tracking in the presence of unresolvable multipath components," IEEE Trans. Vehicular Technology, vol. 48, no. 5, pp. 1573-1583, 1999.

[4] G. Fock, J. Baltersee, P. Schulz-Rittich, and H. Meyr, "Channel tracking for RAKE receivers in closely spaced multipath environments," IEEE Journal on Selected Areas in Communications, vol. 19, no. 12, pp. 2420-2431, 2001.

[5] A. Doucet, J. F. G. de Freitas, and N. J. Gordon, Eds., Sequential Monte Carlo Methods in Practice, Springer, New York, NY, USA, 2001.

[6] J. S. Liu and R. Chen, "Blind deconvolution via sequential imputations," Journal of the American Statistical Association, vol. 90, no. 430, pp. 567-576, 1995.

[7] R. Chen, X. Wang, and J. S. Liu, "Adaptive joint detection and decoding in flat-fading channels via mixture Kalman filtering," IEEE Transactions on Information Theory, vol. 46, no. 6, pp. 2079-2094, 2000.

[8] E. Punskaya, C. Andrieu, A. Doucet, and W. J. Fitzgerald, "Particle filtering for demodulation in fading channels with non-Gaussian additive noise," IEEE Trans. Communications, vol. 49 , no. 4 , pp. 579-582, 2001.

[9] T. Ghirmai, M. F. Bugallo, J. Míguez, and P. M. Djurič, "Joint symbol detection and timing estimation using particle filtering," in Proc. IEEE Int. Conf. Acoustics, Speech, Signal Processing (ICASSP '03), vol. 4, pp. 596-599, Hong Kong, April 2003.

[10] P. M. Djurič, J. H. Kotecha, J. Zhang, et al., "Particle filtering," IEEE Signal Processing Magazine, vol. 2, no. 5, pp. 19-38, September 2003.

[11] Third Generation Partnership Project, "Spreading and modulation (FDD) (Release 1999)," 3G Tech. Spec. (TS) 25.213, v. 3.3.0, Technical Specification Group Radio Access Network, December 2000.

[12] E. Punskaya, A. Doucet, and W. J. Fitzgerald, "On the use and misuse of particle filtering in digital communications," in Proc. 11th European Signal Processing Conference (EUSIPCO '02), Toulouse, France, September 2002.

[13] R. A. Iltis, "A sequential Monte Carlo filter for joint linear/nonlinear state estimation with application to DSCDMA," IEEE Trans. Signal Processing, vol. 51, no. 2, pp. 417426, 2003.

[14] T. Bertozzi, D. Le Ruyet, G. Rigal, and H. Vu-Thien, "On particle filtering for digital communications," in Proc. IEEE Workshop on Signal Processing Advances in Wireless Communications (SPAWC '03), pp. 570-574, Rome, Italy, June 2003.

[15] R. A. Iltis, "Joint estimation of PN code delay and multipath using the extended Kalman filter," IEEE Trans. Communications, vol. 38, no. 10, pp. 1677-1685, 1990.

[16] A. Doucet, S. Godsill, and C. Andrieu, "On sequential Monte Carlo sampling methods for Bayesian filtering," Statistics and Computing, vol. 10, no. 3, pp. 197-208, 2000.

[17] A. J. Viterbi, CDMA: Principles of Spread Spectrum Communication, Addison-Wesley Wireless Communications, Prentice Hall, Englewood Cliffs, NJ, USA, 1995. 
[18] H. Meyr, M. Moeneclaey, and S. Fechtel, Digital Communication Receivers: Synchronization, Channel Estimation, and Signal Processing, Wiley Series in Telecommunications and Signal Processing, John Wiley \& Sons, New York, NY, USA, 2nd edition, 1998.

[19] E. Sourour, G. Bottomley, and R. Ramesh, "Delay tracking for direct sequence spread spectrum systems in multipath fading channels," in IEEE 49th Vehicular Technology Conference (VTC '99), vol. 1, pp. 422-426, Houston, Tex, USA, July 1999.

[20] C. Komninakis, C. Fragouli, A. H. Sayed, and R. D. Wesel, "Multi-input multi-output fading channel tracking and equalization using Kalman estimation," IEEE Trans. Signal Processing, vol. 50, no. 5, pp. 1065-1076, 2002.

[21] P. Tichavsky, C. H. Muravchik, and A. Nehorai, "Posterior Cramer-Rao bounds for discrete-time nonlinear filtering," IEEE Trans. Signal Processing, vol. 46, no. 5, pp. 13861396, 1998.

[22] U. Mengali and A. N. D’Andrea, Synchronization Techniques for Digital Receivers, Plenum Press, New York, NY, USA, 1997.

Tanya Bertozzi received the Dr. Eng. degree in electrical engineering with a specialization in telecommunications from University of Parma, Italy, in 1998. From October 1998 to February 1999, she was a Research Engineer in the Information Department, University of Parma, Italy. In March 1999, she joined Diginext, Aix-en-Provence, France, as a Research Engineer. She is currently a Project Manager. In 2003, she re-

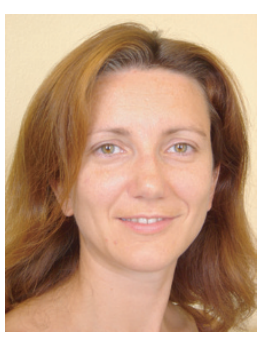
ceived the Ph.D. degree in digital communications from the Conservatoire National des Arts et Métiers (CNAM), Paris, France. Her research interests are in the areas of digital communications and signal processing including particle filtering applications, channel estimation, multiuser detection, and space-time coding.

Didier Le Ruyet received the M.Eng. degree, the M.S. degree, and the Ph.D. degree from the Conservatoire National des Arts et Métiers (CNAM), Paris, France, in 1994 and 2001, respectively. From 1988 to 1995 he was a Research Engineer in the image processing and telecommunication Departments of Sagem, Cergy, France. He joined the Department of Electrical and Computer Engineering at CNAM, Paris, in 1996,

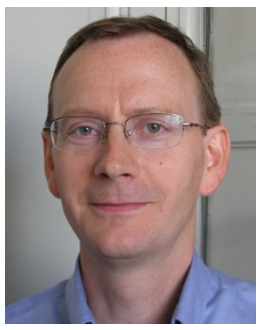
where he became an Assistant Professor in 2002. His main research interests lie in the areas of digital communications and signal processing including channel estimation, channel coding, iterative decoding, and space-time coding.

Cristiano Panazio was born in Brasília, Brazil, in 1977. He received his B.S. and his M.S. degrees in electrical engineering from University of Campinas (UNICAMP), in 1999 and 2001, respectively. Since 2002, he is pursuing a Ph.D. degree at the Conservatoire National des Arts et Métiers (CNAM), Paris, France. His interests include adaptive filtering, synchronization, and nonlinear signal processing.

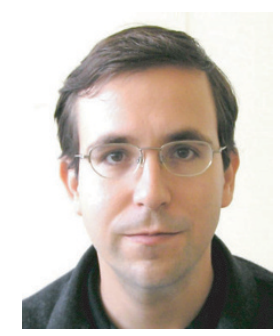

Han Vu Thien received the M. Eng. degree in telecommunications from the École Nationale Supérieure des Télécommunications (ENST), Paris, France, in 1967 and the Ph.D. in physical science from University of Paris XI, Orsay, France, in 1972. He has spent his entire career with the Conservatoire National des Arts et Métiers (CNAM), where he became a Professor of electrical engineering in 1982. Since 1984, he is also

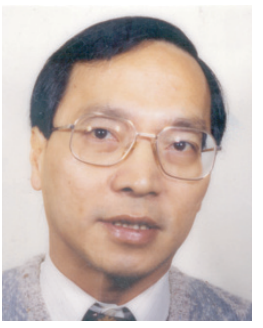
the Director of the Laboratoire des Signaux et systemes. His main research interests lie in image and signal processing for medical applications, Digital television, and HF communication. 